7. E. Snapper, Periodic transformations of affine and projective geometries, Canadian Journal of Mathematics vol. 2 (1950) pp. 149-151.

8. O. Veblen and W. H. Bussey, Finite projective geometries, Trans. Amer. Math. Soc. vol. 7 (1906) pp. 241-259.

Brooklyn College

\title{
NOTE ON LINEAR FORMS
}

\section{J. B. ROBERTS}

1. There has been some interest in solutions to the equation

$$
n=a_{0} x_{0}+a_{1} x_{1}+\cdots+a_{s} x_{8}
$$

where the $a_{i}$ are fixed positive integers with $\operatorname{gcd}=1$ and the $x_{i}$ are non-negative integers. In particular the question of finding the smallest $n$ for which all greater integers have a solution has been investigated to some extent $[1 ; 2]$. It seems that the solution for $s=1$ has been known for some time but that the problem in general remains unsolved for $s>1$. In the paper of $\mathrm{A}$. Brauer cited in the bibliography various upper bounds for the smallest $n$ are given and the actual value of the smallest $n$ is determined for the $a_{i}$ consecutive integers. The main result of this paper is the determination of this smallest $n$ when the $a_{i}$ are in arithmetical progression.

2. Our investigation then is with the linear form

$$
F=a_{0} x_{0}+\cdots+a_{8} x_{8} \text {. }
$$

Throughout this paragraph we assume $2 \leqq a_{0}, \operatorname{gcd} a_{i}=1$ and $a_{j}=a_{0}+j d$. Thus the $a_{i}$ are in arithmetical progression. Then we have the

THEOREM. F represents all $n \geqq N$ where

$$
N=\left(\left[\frac{a_{0}-2}{s}\right]+1\right) \cdot a_{0}+(d-1)\left(a_{0}-1\right)
$$

with non-negative $x_{i}$ and does not so represent $N-1$.

The proof of this result breaks down into a series of five lemmas.

LEMMA 1. The only integers represented by $F$ when $x_{0}+\cdots+x_{s}=m$ are $m a_{0}, m a_{0}+d, m a_{0}+2 d, \cdots, m a_{0}+m s d$.

Proof. $F$ represents $m a_{0}$ for $x_{0}=m$, other $x_{i}=0$. If $F$ represents $m a_{0}+k d$ with $\sum_{0}^{s} x_{i}=m$ and $k<m s$ then $x_{i}>0$ for some $i<s$. In the representation of $m a_{0}+k d$ replace $x_{0}, \cdots, x_{i}, x_{i+1}, \cdots, x_{s}$ by $x_{0}, \cdots, x_{i}-1, x_{i+1}+1, \cdots, x_{s}$. Now $F$ represents $m a_{0}+(k+1) d$.

Received by the editors June $28,1955$. 
Smallest is clearly $m a_{0}$. By induction this gives $m a_{0}+k d$ for $k \leqq m s$. But largest is $m a_{0}+m s d$.

Definition. $[x]^{+}=$the smallest integer $\geqq x$.

Lemma 2 . Let $1 \leqq r \leqq d$ and $j_{r}=\max \left(0,\left[\left(a_{0}-r s-1\right) d s\right]^{+}\right)$. Then all numbers $\geqq\left(r+j_{r} d\right) a_{0}$ which are congruent to r $a_{0}$ modulo $d$ are represented by $F$.

Proof. Every number represented by $F$ has the form $a_{0} \sum_{0}^{s} x_{i}$ $+d \sum_{0}^{s} i x_{i}$. Hence those with the property of being $\equiv r a_{0}(\bmod d)$ are those with $a_{0} \sum_{0}^{s} x_{i} \equiv r a_{0}(\bmod d)$. Since $\left(a_{0}, d\right)=1$ this is equivalent to $\sum_{0}^{s} x_{i} \equiv r(\bmod d)$. Suppose $m=\sum_{0}^{s} x_{i} \equiv r(\bmod d)$. Then $m=r+j d$. Now the largest number represented by $F$ for this $m$ is clearly $m a_{s}$ $=(r+j d)\left(a_{0}+s d\right)$. The next larger number represented by $F$ which is $\equiv r a_{0}(\bmod d)$ is $m^{\prime} a_{0}$ where $m^{\prime}=m+d$. Hence the next larger number will be $(m+d) a_{0}=(r+(j+1) d) a_{0}$. By Lemma 1 we have with $\sum x_{i}=m$ all the numbers $m a_{0}, \cdots, m a_{s}$ and only those represented by $F$. Similarly for $\sum x_{i}=m+d=m^{\prime}$ we have only $m^{\prime} a_{0}, \cdots, m^{\prime} a_{s}$ represented by $F$. The necessary and sufficient condition that there be no numbers which are $\equiv r a_{0}(\bmod d)$ between the largest of $m a_{0}, \cdots, m a_{s}$ which is $m a_{s}=(r+j d)\left(a_{0}+s d\right)$ and the smallest of $m^{\prime} a_{0}, \cdots, m^{\prime} a_{s}$ which is $m^{\prime} a_{0}=(r+(j+1) d) a_{0}$ is that $(r+j d)\left(a_{0}+s d\right)$ $+d \geqq(r+(j+1) d) a_{0}$. This holds if and only if $r s+j s d+1 \geqq a_{0}$. If $r s+1 \geqq a_{0}$ then we can take $j=0=j_{r}$. Otherwise $j \geqq\left(a_{0}-r s-1\right) / d s$ and so the smallest integral value of $j$, denoted by $j_{r}$, is the smallest integer $\geqq\left(a_{0}-r s-1\right) / d s$. Hence $j_{r}=\max \left(0,\left[\left(a_{0}-r s-1\right) / d s\right]^{+}\right)$. Since the condition is necessary and sufficient we shall miss the number $\left(r+j_{r} d\right) a_{0}-d$.

By allowing $r$ to run through the numbers $1,2, \cdots, d$ we shall get $d$ sets of numbers as follows, where $Q_{r}=\left(r+j_{r} d\right) a_{0}$.

$$
Q_{j}, Q_{j}+d, Q_{j}+2 d, \cdots ; \quad j=1,2,3, \cdots, d .
$$

All of these numbers are represented by $F$ by Lemma 2. Also by Lemma 2 we know that $Q_{i}-d$ is not represented by $F$ for any $i=1, \cdots, d$. Since $Q_{i} \equiv i a_{0}(\bmod d)$ we see that any set of $d$ numbers, one from each row, will give a complete set of residues modulo $d$. Hence no two numbers in the above array are the same. Now if we let $Q=\max \left\{Q_{i}\right\}, i=1, \cdots, d$, we see that if $N \geqq Q$ then $N$ is represented by $F$. In fact for $N \geqq Q-d+1$ we have $N$ represented by $F$ and we do not have $Q-d$ represented by $F$. Hence our desired smallest $n$ is just $Q-d+1$.

What remains is to show that $Q-d+1=N$ where $N$ is as given in the theorem. 
Lemma 3. The $Q$ above is equal to $(k+t d) a_{0}$ where

$$
t=\max \left(0,\left[\left(a_{0}-s-1\right) / d s\right]^{+}\right)
$$

and

$$
k=\left\{\begin{array}{l}
d \text { if } t=0 \\
\text { largest of } 1, \cdots, d \quad \text { such that }\left[\left(a_{0}-k s-1\right) / d s\right]+=t \text { if } t \neq 0 .
\end{array}\right.
$$

Proof. $Q=\max \quad\left\{Q_{i}\right\}=\max \quad\left(\left(1+j_{1} d\right) a_{0}, \quad\left(2+j_{2} d\right) a_{0}, \cdots\right.$, $\left.\left(d+j_{d} d\right) a_{0}\right)$. Remembering that $j_{r}=\max \left(0,\left[\left(a_{0}-r s-1\right) / d s\right]^{+}\right)$we see that if $t=0$ then $\left[\left(a_{0}-s-1\right) / d s\right]^{+} \leqq 0$ and hence $\left[\left(a_{0}-r s-1\right) / d s\right]^{+}$ $\leqq 0$ so that $j_{r}=0$ for all $r=1, \cdots, d$. Hence $Q=d a_{0}$. On the other hand if $t \neq 0$ then there is a largest $k$ from 1 to $d$ for which $\left[\left(a_{0}-s-1\right) / d s\right]^{+}=\left[\left(a_{0}-k s-1\right) / d s\right]+$. For the corresponding term of $Q$ we have

$$
\begin{aligned}
\left(k+j_{k} d\right) a_{0} & =\left(k+\left[\left(a_{0}-k s-1\right) / d s\right]+d\right) a_{0} \\
& =\left(k+\left[\left(a_{0}-s-1\right) / d s\right]+d\right) a_{0}=(k+t d) a_{0} .
\end{aligned}
$$

This term is certainly larger than $\left(j+\left[\left(a_{0}-j s-1\right) / d s\right]+d\right) a_{0}$ for $1 \leqq j<k$. Also for $j>k$ we have $\left[\left(a_{0}-j s-1\right) / d s\right]+<\left[\left(a_{0}-k s-1\right) / d s\right]^{+}$ and so $\left(\left[\left(a_{0}-j s-1\right) / d s\right]++1\right) d \leqq\left[\left(a_{0}-k s-1\right) / d s\right]+d=t d$. Hence $\left[\left(a_{0}-j s-1\right) / d s\right]+d \leqq(t-1) d$ so

$\left(j+\left[\left(a_{0}-j s-1\right) / d s\right]+d\right) a_{0} \leqq(j+(t-1) d) a_{0}=((j-d)+t d) a_{0}<(k+t d) a_{0}$.

This completes the proof of the lemma.

LEMMA 4. $F$ represents all numbers $n \geqq N$ where $N=(k+t d) a_{0}$ $-d+1$, and $k, t$ are as defined in Lemma 3, but $F$ does not represent $N-1$.

Proof. This lemma is just a restatement of the remarks preceding Lemma 3 combined with that lemma.

Lemma 5. For $t, k$ defined as in Lemma 3 we have $k+t d=\left[\left(a_{0}-2\right) / s\right]$ $+d$.

Proof. (a) $t=0$. By definition of $t$ we see that $\left[\left(a_{0}-s-1\right) / d s\right]+\leqq 0$ so $s+1 \geqq a_{0}$ and $a_{0}-2 \leqq s-1<s$. Therefore $\left[\left(a_{0}-2\right) / s\right]=0$. Also, by definition of $k$ we have $k=d$. Hence $k+t d=d=\left[\left(a_{0}-2\right) / s\right]+d$.

(b) $t \neq 0$. Suppose that

$$
\frac{a_{0}-s-1}{d s}=I+\frac{j}{d s}, \quad I \text { an integer, } 0 \leqq j<d s .
$$


Then

$$
\frac{a_{0}-k s-1}{d s}=I+\frac{j}{d s}-\frac{k-1}{d}
$$

and

$$
\frac{a_{0}-(k+1) s-1}{d s}=I+\frac{j}{d s}-\frac{k}{d} .
$$

Also by definition of $k$ we know

$$
\left[\frac{a_{0}-s-1}{d s}\right]^{+}=\left[\frac{a_{0}-k s-1}{d s}\right]^{+}>\left[\frac{a_{0}-(k+1) s-1}{d s}\right]^{+} .
$$

We now have two cases; first with $j=0$ and secondly with $j \neq 0$.

(i) $j=0$. From (1), (2), and (4) we see that $(k-1) / d<1$ or $k<d+1$. Also from (1), (3), and (4) we have $k \geqq d$. Hence $k=d$. Now $\left[\left(a_{0}-2\right) / s\right]$ $=\left[\left(a_{0}-s-1\right) / s+(1-1 / s)\right]=\left(a_{0}-s-1\right) / s$ since $\left(a_{0}-s-1\right) / s=d I$ which is an integer while $(1-1 / s)<1$. Thus $k+t d=d$ $+\left[\left(a_{0}-s-1\right) / d s\right]+d=\left[\left(a_{0}-2\right) / s\right]+d$.

(ii) $j \neq 0$. From (1)-(4) we deduce that $j / d s-(k-1) / d>0$ and $j / d s-k / d \leqq 0$. Hence $k<j / s+1$ and $k \geqq j / s$. Therefore $k=[j / s]+$. Now $\left[\left(a_{0}-s-1\right) / d s\right]^{+}=[I+j / d s]^{+}=I+1=\left(a_{0}-s-1\right) / d s-j / d s+1$. Hence $k+t d=k+\left[\left(a_{0}-s-1\right) / d s\right]+d=[j / s]++\left(a_{0}-s-1\right) / s-j / s+d$. Now either $j / s$ is an integer or not. We suppose first that it is an integer. Then $[j / s]^{+}=j / s$. Since $I=\left(a_{0}-s-1\right) / d s-j / d s$ we have $\left(a_{0}-s-1\right) / s$ $=d I+j / s$ which is an integer. Thus $\left[\left(a_{0}-2\right) / s\right]=\left[\left(a_{0}-s-1\right) / s\right.$ $+(1-1 / s)]=\left(a_{0}-s-1\right) / s$ and $k+t d=\left(a_{0}-s-1\right) / s+d=\left[\left(a_{0}-2\right) / s\right]$ $+d$. We now suppose that $j / s$ is not an integer but that it is equal to $J+i / s$ where $J$ is an integer and $0<i<s$. In this case $[j / s]^{+}=J+1=j / s-i / s+1$. Therefore $k+t d=[j / s]++\left(a_{0}-s-1\right) / s$ $-j / s+d=1-i / s+\left(a_{0}-s-1\right) / s+d$. Now $\quad\left(a_{0}-s-1\right) / s=d I+j / s$ $=d I+J+i / s$ so $\left[\left(a_{0}-2\right) / s\right]=\left[\left(a_{0}-s-1\right) / s+(1-1 / s)\right]=[d I+J$ $+(i-1) / s+1]$. Since $1 \leqq i \leqq s-1$ we know $0 \leqq(i-1 / s)<1$. Therefore $\left[\left(a_{0}-2\right) / s\right]=d I+J+1=\left(a_{0}-s-1\right) / s-i / s+1=k+t d-d$ and so $\left[\left(a_{0}-2\right) / s\right]+d=k+t d$. This completes the proof of the lemma.

Putting Lemmas 4 and 5 together gives us the proof of the theorem.

3. In $\$ 2$ we have disposed of the problem of $\$ 1$ when the $a_{i}$ are in arithmetic progression. The simplest case not covered seems to be that where the $a_{i}$ are general but $s=2$. Even this case seems quite difficult however. We state the following result which is a specialization of the case just mentioned.

Given $F=a x_{0}+(a+1) x_{1}+(a+z) x_{2}, z>2$, and the $x_{i}$ to be nonnegative then the smallest $N$ for which all $n \geqq N$ are represented by $F$ but with $N-1$ not so represented is given by 
(a) $((a+1) / z) a+(z-3) a \quad$ when $a \equiv z-1(\bmod z)$ and $a \geqq z^{2}-5 z+3$,

(b) $[(a+1) / z](a+z)+(z-3) a$

when $a \not \equiv z-1(\bmod z)$ and $a \geqq z^{2}-4 z+2$.

We omit the proof of this result as it is rather long.

It is not hard to find the desired $N$ for specific triples of numbers. For instance when $a_{0}, a_{1}=a_{0}+2, a_{2}=a_{0}+3$ we find the value of $N$ to be $[x / 3] \cdot x+2+x$. If the largest of $a_{0}, a_{1}, a_{2}$ is sufficiently larger than the other two and those two are relatively prime then the $N$ is easily determined also. In fact if $a_{0}<a_{1}<a_{2}$ and $\left(a_{0}, a_{1}\right)=1$ and $a_{2}>\left(a_{0}-1\right)\left(a_{1}-1\right)-a_{0}$ then $N=\left(a_{0}-1\right)\left(a_{1}-1\right)$.

\section{BIBLIOGRAPHY}

1. A. Brauer, On a problem of partitions, Amer. J. Math. vol. 64 (1942) pp. 299312.

2. J. B. Roberts, Interaction of cycles, Bulletin of Mathematical Biophysics vol. 10 (1948) pp. 123-129.

\section{Reed College}

\section{ON THE INFINITUDE OF PRIMITIVE $k$-NONDEFICIENTS}

\section{HERBERT A. BERNHARD}

H. N. Shapiro has defined [1] a $k$-nondeficient as an integer $n$ which satisfies

$$
\sigma(n) / n \geqq k
$$

where $\sigma(n)$ is the sum of the divisors of $n$. Integers $n$ which do not satisfy (1) are called $k$-deficient. A primitive $k$-nondeficient is defined as a $k$-nondeficient, all of whose proper divisors are $k$-deficient. In the same paper, Shapiro shows that, in order for an infinite number of primitive $k$-nondeficients to exist, it is necessary that $k$ be of the form

$$
\prod_{i=1}^{m} \frac{p_{i}^{\alpha_{i}+1}-1}{\left(p_{i}-1\right) p_{i}^{\alpha_{i}}} \prod_{i=m+1}^{n} \frac{p_{i}}{p_{i}-1}
$$

or, written another way,

$$
\prod_{i=1}^{m} \frac{\sigma\left(p_{i}^{\alpha_{i}}\right)}{p_{i}^{\alpha_{i}}} \prod_{i=m+1}^{n} \frac{p_{i}}{p_{i}-1}
$$

where $p_{1}, p_{2}, p_{3}, \cdots, p_{n}$ are distinct primes and $0 \leqq m \leqq n$. In this note we show that, for every $k$ of the form (2), an infinite number of

Received by the editors May 26, 1955. 